Article

\title{
An Off-Axis Differential Method for Improvement of a Femtosecond Laser Differential Chromatic Confocal Probe
}

\author{
Chong Chen, Yuki Shimizu *(D), Ryo Sato, Hiraku Matsukuma and Wei Gao
}

Precision Nanometrology Laboratory, Department of Finemechanics, Tohoku University, Sendai 980-8579, Japan; chongchen@nano.mech.tohoku.ac.jp (C.C.); satoryo@nano.mech.tohoku.ac.jp (R.S.);

hiraku.matsukuma@nano.mech.tohoku.ac.jp (H.M.); gaowei@cc.mech.tohoku.ac.jp (W.G.)

* Correspondence: yuki.shimizu@nano.mech.tohoku.ac.jp; Tel.: +81-22-795-6950

Received: 8 September 2020; Accepted: 15 October 2020; Published: 16 October 2020

\begin{abstract}
This paper presents an off-axis differential method for the improvement of a femtosecond laser differential chromatic confocal probe having a dual-detector configuration. In the proposed off-axis differential method employing a pair of single-mode fiber detectors, a major modification is made to the conventional differential setup in such a way that the fiber detector in the reference detector is located at the focal plane of a collecting lens but with a certain amount of off-axis detector shift, while the fiber detector in the measurement detector is located on the rear focal plane without the off-axis detector shift; this setup is different from the conventional one where the difference between the two confocal detectors is provided by giving a defocus to one of the fiber detectors. The newly proposed off-axis differential method enables the differential chromatic confocal setup to obtain the normalized chromatic confocal output with a better signal-to-noise ratio and approaches a Z-directional measurement range of approximately $46 \mu \mathrm{m}$, as well as a measurement resolution of $20 \mathrm{~nm}$, while simplifying the optical alignments in the differential chromatic confocal setup, as well as the signal processing through eliminating the complicated arithmetic operations in the determination of the peak wavelength. Numerical calculations based on a theoretical equation and experiments are carried out to verify the feasibility of the proposed off-axis differential method for the differential chromatic confocal probe with a mode-locked femtosecond laser source.
\end{abstract}

Keywords: chromatic confocal probe; femtosecond laser; off-axis differential method; tracking local minimum method

\section{Introduction}

A confocal probe is an optical displacement sensor often employed in microscopy for form measurement of three-dimensional microstructures for the quality control of ultra-precision machined surfaces [1-6]. Due to a high lateral resolution and a good depth discrimination performance by the Z-directional depth-sectioning effect, a confocal probe can also be employed in the surface profilometry of various materials and medical applications in the observation of living cells [7-10]. Chromatic confocal microscopy [11] has been proposed for the elimination of the axial-scanning of the conventional confocal microscopy. To obtain a better lateral resolution and an in-depth discrimination performance while eliminating the influence of nonuniform surface reflectivity of a target object in the three-dimensional surface topography measurement at nanometric or sub-nanometric level, differential methods and the corresponding signal processing algorithms for a confocal probe [12-26] have been proposed. Most of the differential methods for a confocal probe employ two confocal outputs captured by two detectors with different apertures or focusing conditions. 
The differential methods can be categorized into two major types. The first type [25] employs two photodetectors having apertures with different sizes. Each of the apertures is placed at the focal plane of the corresponding collecting lens in the confocal setup, and the height information of an object surface under inspection can be decoded from the linear relationship between the axial displacement of the target surface and the normalized intensity ratio of the two confocal outputs. Some of the differential probes of this type can carry out measurement without the scanning along the optical axis. However, the in-depth measurement range is relatively small [26].

The second type employs two detectors having identical confocal apertures [17-24]. One confocal aperture is located in front of the focal plane of a corresponding collecting lens, while the other is placed behind the focal plane of another corresponding collecting lens to obtain two different confocal outputs. In the case of employing two slits as the apertures for the confocal setups, the in-depth resolution could be degraded [17]. However, this can be overcome by employing two identical pinholes as the apertures for the confocal setup [18-24]. For this type of differential method, two corresponding signal processing algorithms have been designed. The first signal processing algorithm [21-24] is designed in such a way that the normalized output is defined as the ratio of intensity subtraction of the two confocal outputs. Meanwhile, this algorithm cannot remove the influence of the nonuniform surface reflectivity of an object under inspection, as well as the influence of the light source. To address these issues, a second signal processing algorithm [18-20] has been designed. In the algorithm, the ratio of intensity difference and intensity sum (or maximum intensity) of the two confocal outputs are utilized as a confocal output. It should be noted that these two types can be combined with each other [26].

In the previous work by the authors of [27-31], the dual-detector confocal setup based on the second type, as well as the signal processing algorithm, has been modified for the femtosecond laser differential chromatic confocal probe, where a mode-locked femtosecond laser is employed as the laser source. In the modified differential chromatic confocal setup, the two identical fiber detectors are arranged in such a way that the fiber detector in the measurement detector unit is placed at the focal plane of the collecting lens of the detector unit, as shown in Figure 1a, while the fiber detector in the reference detector unit is placed at a position with a displacement (defocus) $d$ along with the axial position, as shown in Figure $1 \mathrm{~b}$. For acquiring the in-depth information, two confocal outputs with these detectors in different confocal setups are utilized. For the signal processing, the algorithm referred to as the tracking local minimum (TLM) method has been proposed [27]. In the TLM method, the normalized confocal output is defined as the ratio of the measurement detector output to the reference detector output. This algorithm can theoretically eliminate the influences of the nonuniform spectrum of a mode-locked femtosecond laser source. Meanwhile, the intensity around the first local minimum of the reference detector output spectrum is relatively small, and hence the normalized output can easily be influenced by out-of-focus noise [25,32-34].

(a)

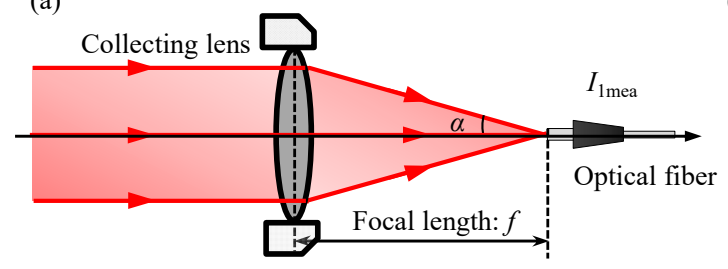

(b)

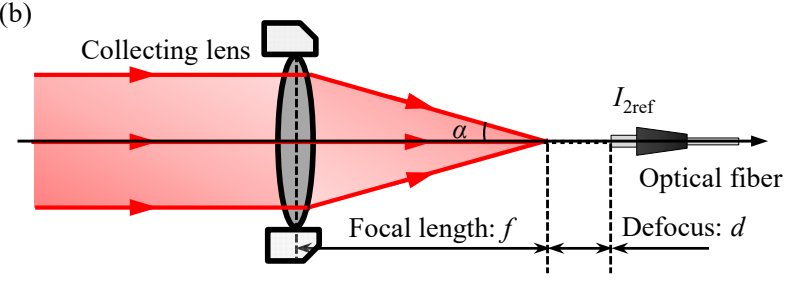

Figure 1. Schematics of the relative position between the fiber core and the collecting lens in measurement and reference detectors in the femtosecond confocal probe. (a) A setup for the measurement detector. (b) A setup for the reference detector with a defocus $d$.

To address the issue, in this paper, a major modification is made to the conventional differential chromatic confocal setup in the mode-locked femtosecond laser differential chromatic confocal probe. Figure 2 shows a schematic of the confocal setup of the reference detector unit employed in the newly proposed method. As can be seen in the figure, the fiber detector is placed at the focal plane of 
the collecting lens while an off-axis detector shift $d_{\mathrm{m}}$ is given to the fiber detector, instead of giving a defocus $d$; this modification improves the signal-to-noise ratio of the normalized chromatic confocal output to be obtained in the differential confocal setup. This optical configuration is also expected to make the optical alignment of the differential confocal setup easier than the conventional setup shown in Figure 1. Theoretical analysis and experimental examination are implemented to verify the feasibility of the proposed off-axis differential method for the differential chromatic confocal probe with a mode-locked femtosecond laser source. It should be noted that the work described in this paper is mainly intended to focus on the proposal of the preliminary idea of an off-axis differential method for the improvement of the signal-to-noise ratio (SNR), the measurement range and the measurement resolution of the femtosecond laser chromatic confocal probe. The subsequent application of this new method is needed in the imaging of three-dimensional (3D) microstructures in our future work. Regarding the spectral range (from 1480 to $1640 \mathrm{~nm}$ ) and the chromatic objective lens employed in the experiments, a measurement range of $46 \mu \mathrm{m}$ is expected to be achieved through the above improvements by the proposed off-axis differential method.

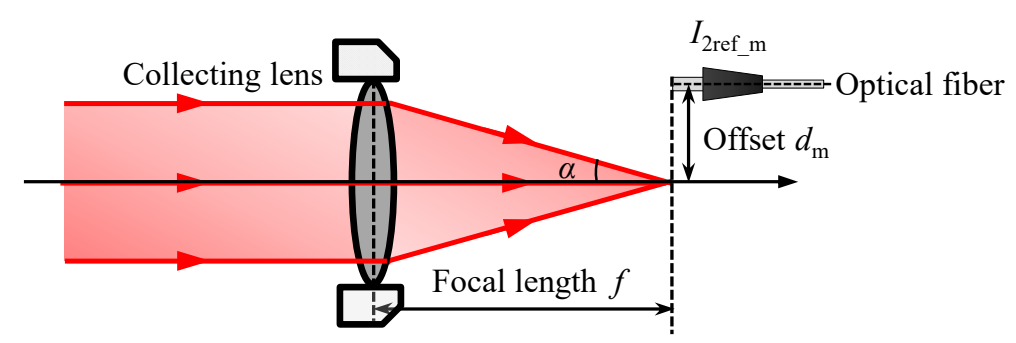

Figure 2. Reference detector unit with an off-axis detector shift $d_{\mathrm{m}}$ in the newly proposed method.

\section{Measurement Principle}

\subsection{Image Formation Principle}

Figure 3 shows a schematic diagram of the differential chromatic confocal configuration [27-31]. A mode-locked femtosecond laser beam from the laser source is made to focus on a target surface by a chromatic objective lens. The reflected laser beam from the target surface is then divided into two sub-beams by a beam splitter, and the sub-beams are then captured by two fiber detector units composed of two identical fibers and two identical collecting lenses, $\mathrm{L}_{1}$ and $\mathrm{L}_{2}$. One of the sub-beams is captured by one fiber detector unit (referred to as the measurement detector unit) set at the focal plane of $\mathrm{L}_{1}$, while the other is captured by another fiber detector unit (referred to as the reference detector unit) with the collecting lens $\mathrm{L}_{2}$. 


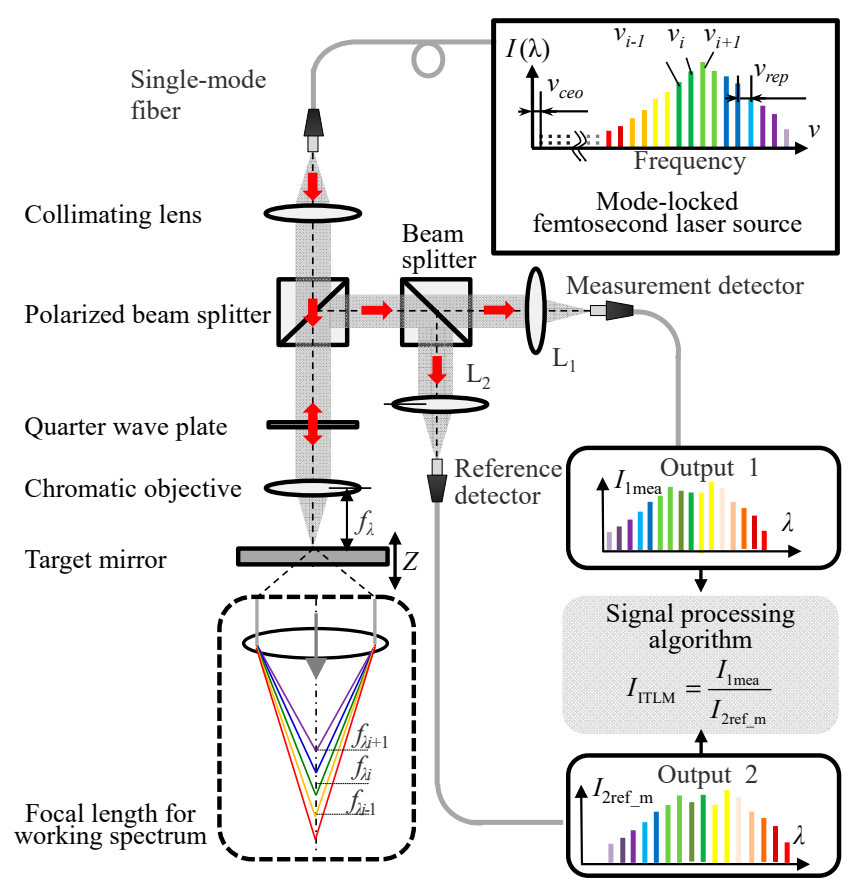

Figure 3. A schematic diagram of the differential chromatic confocal configuration with a mode-locked femtosecond laser source.

The confocal output $I_{1 \text { mea }}(u)$ to be captured by the measurement detector unit, whose optical configuration is shown in Figure 1a, can be expressed by the following equation [27,35-37].

$$
I_{1 \text { mea }}(u)=\left|\int_{0}^{1} \exp \left(j u \rho^{2}\right) P_{1 \text { eff }}(\rho) P_{2 \text { eff }}(\rho) \rho d \rho\right|^{2}
$$

where $\rho$ is the normalized radius of the imaging lens, $j$ is the imaginary unit and $P_{1 \text { eff }}$ and $P_{2 \text { eff }}$ stand for the effective pupil functions of the imaging lens (L1 or L2) and illuminating lens (collimating lens), respectively. The parameters $v$ and $u$ represent the optical coordinates related to the actual radial and axial coordinates $r$ and $z$, respectively, that can be given as follows [27,35-37]. It should be noted that only the axial (Z-) response is considered and measured in the experiments. Therefore, the variable $\mathrm{v}$ in Equation (1) is treated to be zero [27] and is omitted in the equation.

$$
\begin{gathered}
v=\frac{2 \pi}{\lambda} r \sin \beta \\
u=\frac{2 \pi}{\lambda} z \sin ^{2} \beta
\end{gathered}
$$

where $\sin \beta$ and $\lambda$ are the numerical aperture of the collecting lens and the light wavelength, respectively.

Figure 4 shows schematic diagrams of the reference fiber detector unit by the proposed off-axis differential method and the conventional differential method for the chromatic confocal probe system. As can be seen in Figure $4 a$, in the reference fiber detector unit based on the conventional differential method [27], the single-mode fiber detector is placed with a defocus $d$ with respect to the back focal plane of the collecting lens $\mathrm{L}_{2}$. In this case, the confocal output $I_{2 \text { ref }}\left(u ; u_{\mathrm{d}}\right)$ can be represented as follows:

$$
I_{2 \operatorname{ref}}\left(u ; u_{d}\right)=\left|\int_{0}^{1} \exp \left[j\left(u+u_{d} / 2\right) \rho^{2}\right] P_{1 \mathrm{eff}}(\rho) P_{2 \mathrm{eff}}(\rho) \rho d \rho\right|^{2}
$$


(a)

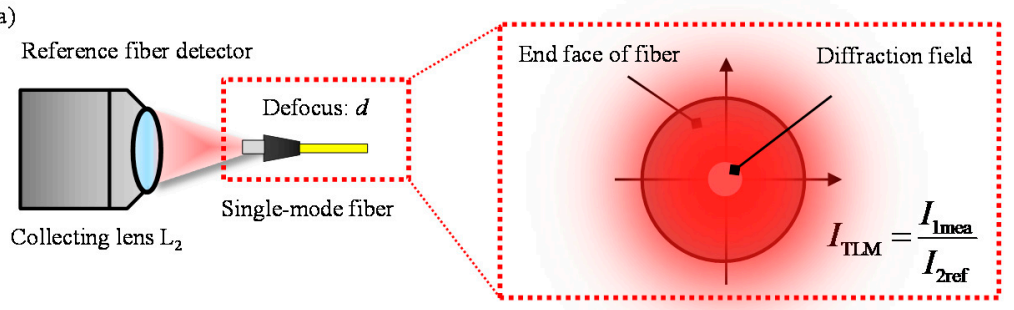

(b)

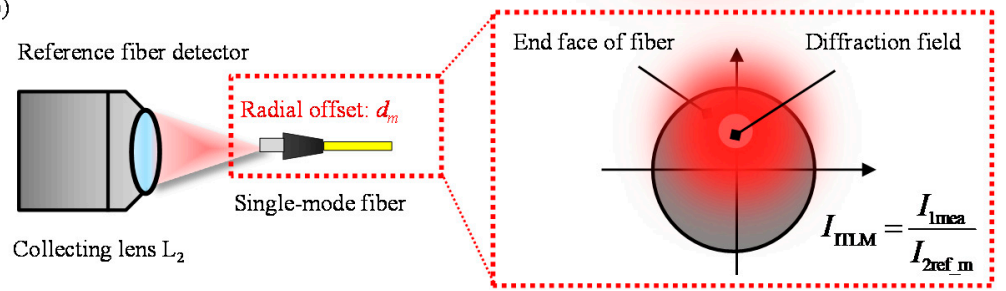

Figure 4. Schematic of the reference fiber detector settings by the proposed off-axis differential method and conventional differential method. (a) Schematic diagram of the reference fiber detector setting by the conventional differential method; (b) schematic diagram of the reference fiber detector setting by the proposed off-axis differential method.

By using these two confocal outputs, the height of a surface under measurement can be detected through the arithmetic operation based on the algorithm referred to as the tracking local minimum (TLM) method [30]. Meanwhile, in the newly proposed off-axis differential method, a major modification is made to the reference detector unit: the single-mode fiber detector is located on the rear focal plane of the collecting lens $\mathrm{L}_{2}$ by the optical axis but with an off-axis detector shift $d_{\mathrm{m}}$, as shown in Figure $4 \mathrm{~b}$. In this case, the confocal output $I_{2 \text { ref_m }}\left(u ; d_{\mathrm{m}}\right)$ can be represented as follows [38]:

$$
I_{2 \text { ref_m }}\left(u, d_{\mathrm{m}}\right)=\left|\int_{0}^{1} J_{0}\left(\frac{d_{\mathrm{m}}}{r_{\mathrm{f}}} \rho v_{\mathrm{f}}\right) \exp \left(j u \rho^{2}\right) P_{1 \text { eff }} P_{2 \operatorname{eff}} \rho d \rho\right|^{2}
$$

where $r_{\mathrm{f}}$ and $v_{\mathrm{f}}$ are the radius and the normalized radius of the single-mode fiber, respectively. As can be seen in Equations (4) and (5), the confocal output becomes different in the modified setup. According to Equation (5), the effect of employing the proposed off-axis differential method is similar to reducing the core diameter of a fiber detector acting as a pinhole aperture in the confocal setup; namely, the rejection of more scattered light and out-of-focus noise in the reference fiber detector can be realized. This effect is expected to improve the signal-to-noise $(\mathrm{S} / \mathrm{N})$ ratio of the confocal output, as well as the resolution, in the differential chromatic confocal probe.

\subsection{Signal Processing Algorithm}

In the tracking local minimum (TLM) algorithm [30], the normalized chromatic output $I_{\mathrm{TLM}}$ is defined as the intensity ratio of the two fiber detector outputs [27-30]. Denoting the chromatic output

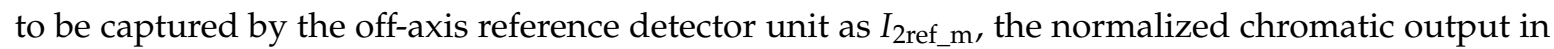
the newly proposed off-axis differential method can be expressed as follows:

$$
I_{\operatorname{ITLM}}\left(u, d_{m}\right)=\frac{I_{1 \mathrm{mea}}(u)}{I_{2 \text { ref_m }}\left(u, d_{m}\right)}
$$

Figure 5a shows a graphical diagram of the TLM algorithm [30]. The wavelength at a specific peak position of the normalized chromatic output $I_{\text {ITLM }}$, which corresponds to the wavelength at the first local minimum of the confocal output $I_{2 \text { ref_m, }}$ changes linearly with respect to the Z-directional displacement of the target surface. A displacement of the target mirror can thus be acquired by tracking the wavelength at the first local minimum of the confocal output through detecting the peak wavelength in the normalized chromatic output $I_{\text {ITLM }}$. Figure $5 b$ shows a schematic of 
the wavelength-to-displacement encoding by the signal processing algorithm. In the tracking local minimum algorithm [27], the asymmetric peak in the normalized chromatic output $I_{\text {ITLM }}$ could make it difficult under practical conditions to precisely determine the peak wavelength due to the out-of-focus noise [30]. To address the issue, a centroid wavelength of the peak is employed as the peak wavelength.

(a)

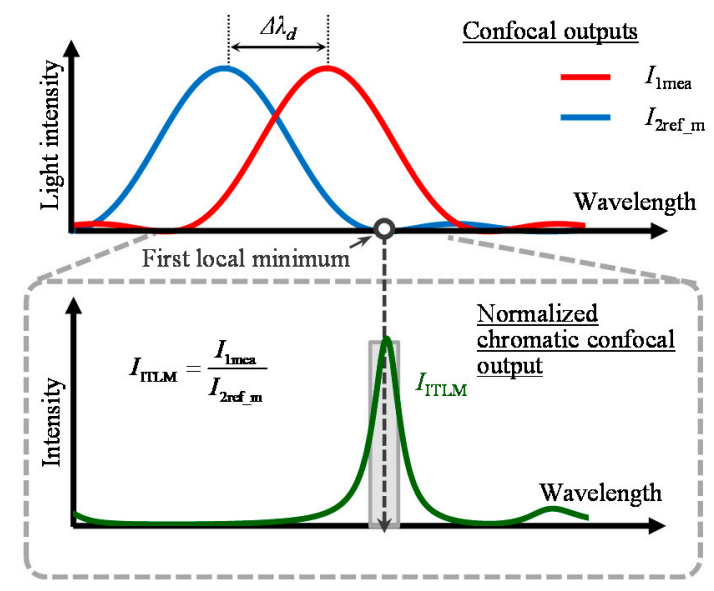

(b)
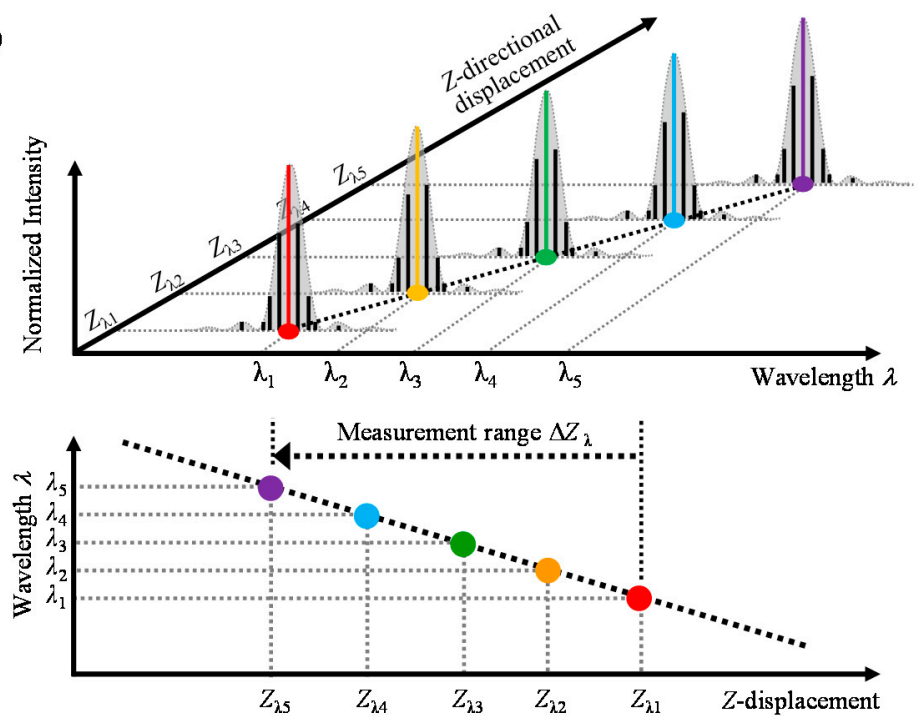

Figure 5. A schematic of the tracking local minimum (TLM) algorithm. (a) The normalized output obtained from the reference output and the measurement output [30]; (b) a schematic of the wavelength-to-displacement encoding in the TLM method.

\section{Experiments and Discussions}

\subsection{Experimental Confocal Configuration}

To verify the feasibility of the proposed off-axis differential method, experiments were carried out by using the developed differential chromatic confocal system, a schematic diagram of which is shown in Figure 6. For a fair comparison, the optical components in the configuration are the same as those adopted in the previous work by the authors of [30] based on the defocus setup, except a manual stage for the adjustment of the off-axis detector shift $d_{\mathrm{m}}$ of the fiber detector in the reference fiber detector unit. In this paper, $d_{m}$ in the positive $Y$-direction was treated to be positive. 


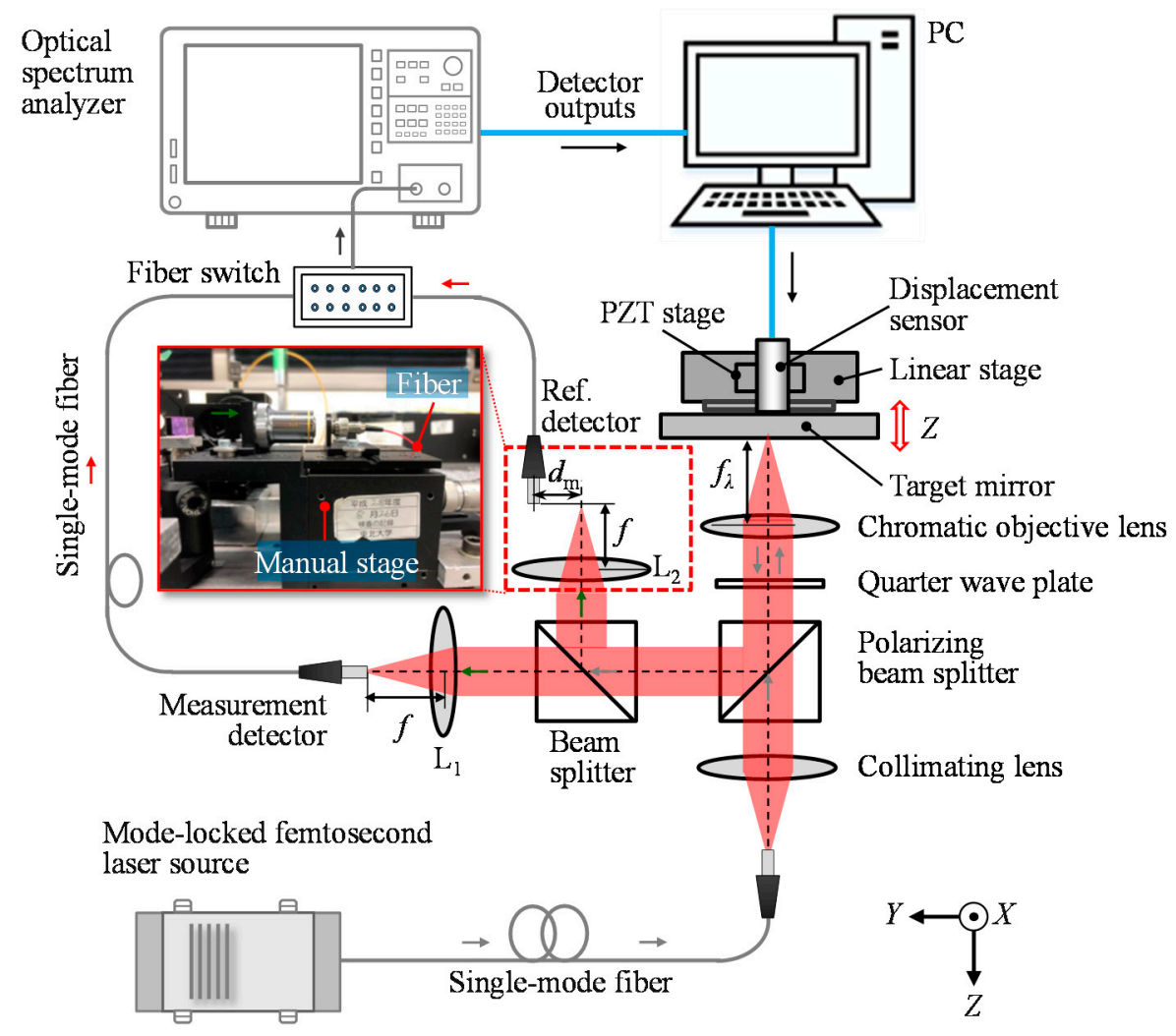

Figure 6. A schematic diagram of the proposed off-axis differential chromatic confocal probe configuration with a mode-locked femtosecond laser source [30].

At first, the coarse adjustment of the off-axis fiber in the reference detector was carried out, since too much offset $d_{\mathrm{m}}$ could result in the local minimum in the reference detector output out of the wavelength range of the optical spectrum analyzer employed in the setup. The amount of $d_{\mathrm{m}}$ given to the fiber detector in the reference detector unit was adjusted so that the first local minimum wavelength of the confocal output $I_{2 \text { ref_m }}$ could be captured at each Z-directional position of a target surface. It should be noted that the single-mode fiber (SMF-28e, Corning Inc.) had a core diameter of $8.2 \mu \mathrm{m}$ and an NA of 0.14 . The detector was composed of a single-mode fiber and an optical spectrum analyzer. At each Z-position, it took about $8 \mathrm{~s}$ to capture the spectrum by the measurement and reference detectors. A bandwidth of the optical spectrum analyzer was set to be $0.5 \mathrm{~nm}$ with a sampling interval of $0.1 \mathrm{~nm}$. Figure 7 shows the noise reduction performances in the comparison between the conventional defocus differential method and the proposed off-axis differential method. As can be seen in the figures, the out-of-focus noises at the ends of the measurement range (positions A1 and B1) could be found in the conventional defocus differential method. Meanwhile, the out-of-focus noises at the ends of the measurement range (positions A2 and B2) were found to be reduced in the newly proposed off-axis differential method; this contributes to achieving a wider measurement range. These results mean that a better signal-to-noise ratio (SNR) of the normalized chromatic output IILM can be expected by the newly proposed off-axis differential method. It should be noted that this can also contribute to carrying out stable detection of the peak wavelength to be employed for the detection of the displacement of a surface under inspection through the wavelength-to-displacement encoding. 
(a)
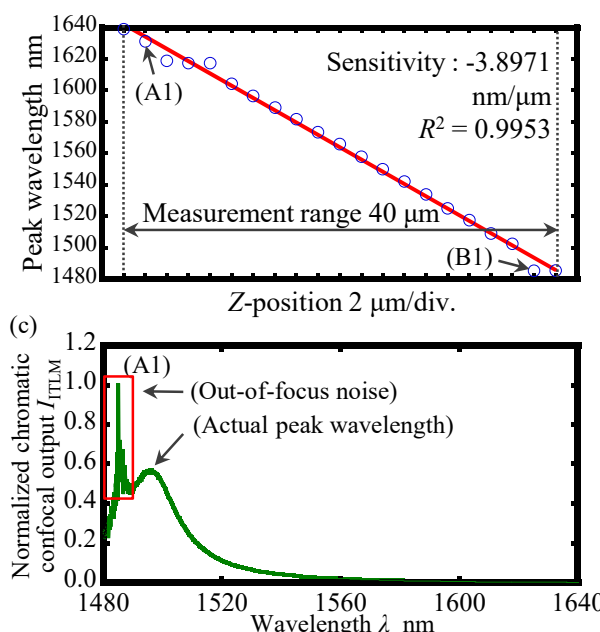

(e)

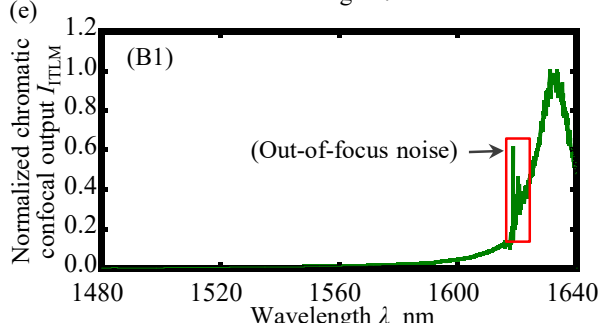

(b)
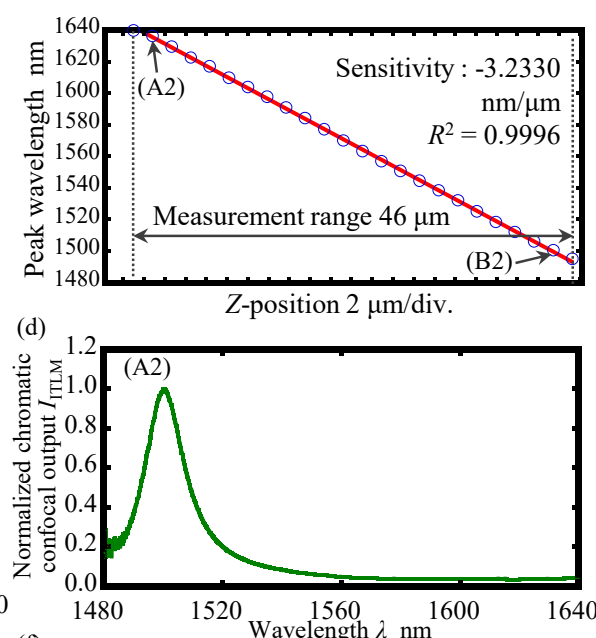

(f)

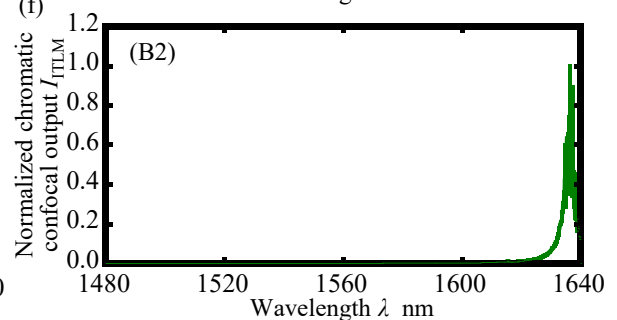

Figure 7. Noise reduction performances in the two methods. (a) Measurement range of the conventional defocus differential method; (b) measurement range of the proposed off-axis differential method; (c) normalized chromatic confocal output ITTLM in (A1) position; (d) normalized chromatic confocal output $I_{\text {ITLM }}$ in (A2) position; (e) normalized chromatic confocal output $I_{\text {ITLM }}$ in (B1) position; (f) normalized chromatic confocal output $I_{\text {ITLM }}$ in (B2) position.

Figure 8a shows the spectrum of the mode-locked femtosecond laser employed in the experiments, and Figure $8 \mathrm{~b}$ shows the typical spectra of the two confocal outputs, $I_{1 \mathrm{mea}}$ and $I_{2 \text { ref_m }}$, captured by the dual-fiber detector units. As can be seen in Figure $8 \mathrm{~b}$, due to the nonuniform spectrum of the utilized mode-locked femtosecond laser source shown in Figure 8a, it is not so easy to figure out peak wavelengths in the obtained spectra. Figure $8 \mathrm{c}$ shows the normalized chromatic confocal output $I_{\text {ITLM }}$ obtained from the spectra shown in Figure 8a,b. As can be seen in Figure 8c, it is much easier to figure out the peak in the spectrum with a much smaller full width at half maximum (FWHM) value. 
(a)

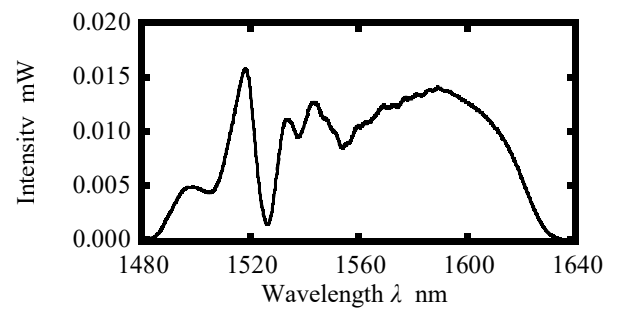

(b)

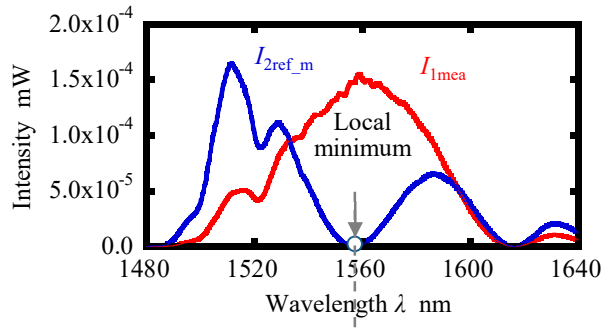

(c)

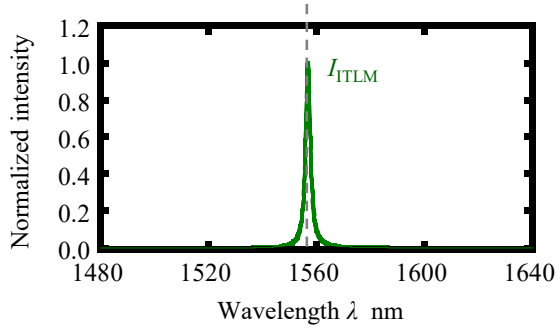

Figure 8. Schematic of the wavelength-to-displacement encoding in the proposed off-axis differential chromatic confocal probe system. (a) A spectrum of the mode-locked femtosecond laser employed in this paper; (b) chromatic confocal outputs obtained by the detector units; (c) the normalized chromatic confocal output obtained through the arithmetic operation with $I_{1 \text { mea }}$ and $I_{2 \text { ref_m }}$.

\subsection{Optimum of the Misalignment Value}

The measurement resolution of the femtosecond laser differential chromatic confocal probe based on the TLM algorithm could be affected by the sharpness of the peak in the normalized chromatic confocal output [30]. In this paper, the appropriate off-axis detector shift $d_{\mathrm{m}}$ was investigated through experiments by using the full width at half maximum (FWHM) of the peak in the normalized chromatic confocal output $I_{\text {ITLM }}$. At first, numerical calculations [28] were carried out based on Equation (5) to estimate the variation of the FWHM due to the change in $d_{\mathrm{m}}$. Figure 9 shows the result. As can be seen in the figure, the FWHM was estimated to reduce as there was an increase in the off-axis displacement $d_{\mathrm{m}}$. It should be noted that the simulation based on Equation (5) is valid under the ideal condition of a simplified optical configuration without any aberrations.

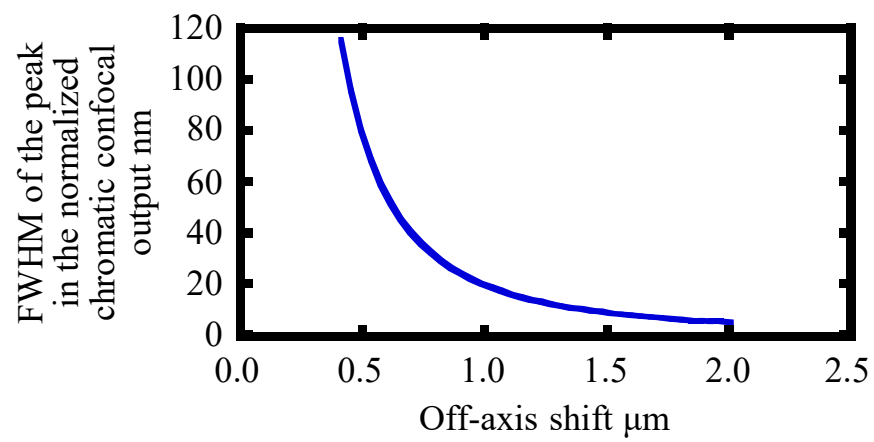

Figure 9. Variation of the full width at half maximum (FWHM) of the peak in the normalized chromatic confocal output estimated by the numerical calculations. 
Following the numerical calculations, experiments were carried out. In the experiments, the central wavelength $\lambda_{0}$ was set to be approximately $1560 \mathrm{~nm}$. The off-axis displacement $d_{\mathrm{m}}$ of the fiber detector in the reference detector was changed in a step of $1 \mu \mathrm{m}$, while obtaining the normalized chromatic confocal output at each $d_{\mathrm{m}}$. Figure 10 shows the results. As can be seen in the figure, the FHWM of the peak in $I_{\text {ITLM }}$ was found to deviate with the change in $d_{\mathrm{m}}$. Figure 11 summarizes the variation of the FWHM observed in Figure 10. As can be seen in the figures, FWHM values can clearly be figured out when the mismatch displacement (off-axis shift) is smaller than $3 \mu \mathrm{m}$. Due to the effects of the numerical aperture (mismatch) on the normalized chromatic confocal output $I_{\text {ITLM }}$, it becomes difficult to find out the FWHM value for the case having large $d_{\mathrm{m}}$. The FWHM value was found to be minimized around $d_{\mathrm{m}}=1 \mu \mathrm{m}$. The root cause of the difference observed between the results of numerical calculations and the experiments is not clear, and further investigation will be carried out in future work. Regarding the experimental results, $d_{\mathrm{m}}$ was set to be $1 \mu \mathrm{m}$ in the following experiments. It should be pointed out that the determination of the appropriate off-axis detector shift $d_{\mathrm{m}}$ is a less time-consuming task compared with the determination of the appropriate defocus in the conventional setup, where the defocus $d$ needs to be changed in a wide range up to hundreds of micrometers depending on the confocal setup; this is another advantage of the newly proposed off-axis differential method compared with the conventional defocus method.

(a)

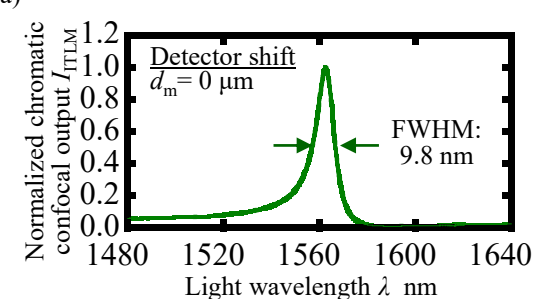

(c)

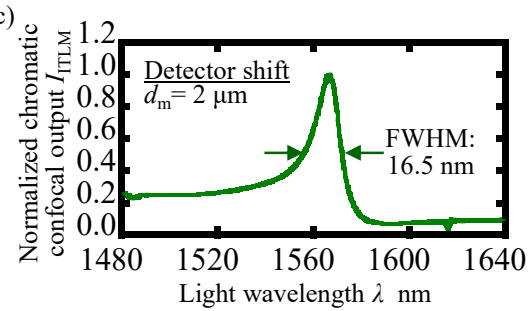

(b)

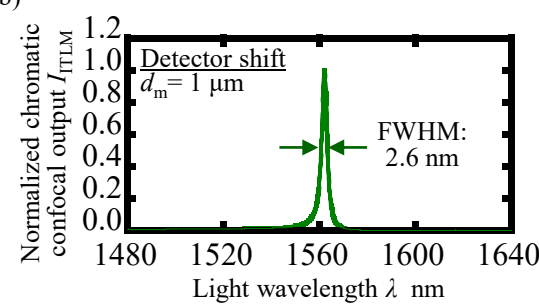

(d)

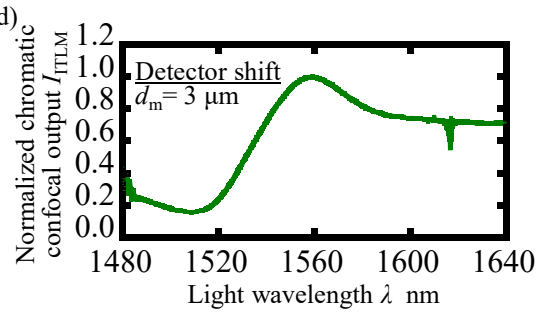

Figure 10. Normalized chromatic confocal output at each off-axis displacement $d_{\mathrm{m}}$ of the fiber detector in the reference detector. (a) $d_{\mathrm{m}}=0 \mu \mathrm{m}$; (b) $d_{\mathrm{m}}=1.0 \mu \mathrm{m}$; (c) $d_{\mathrm{m}}=2.0 \mu \mathrm{m}$; (d) $d_{\mathrm{m}}=3.0 \mu \mathrm{m}$.

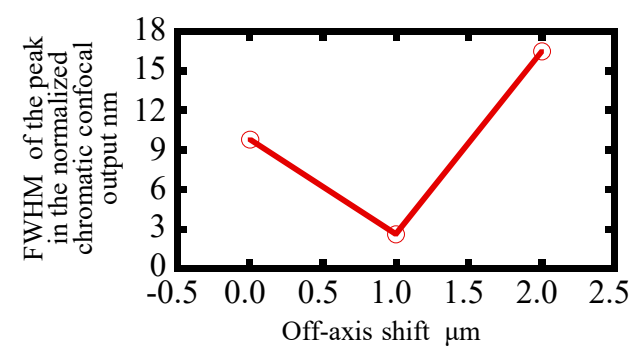

Figure 11. Variation of the FWHM of the peak in the normalized chromatic confocal output due to the change in the off-axis displacement $d_{\mathrm{m}}$ of the fiber detector in the reference detector.

\subsection{Evaluation of the Measurement Range and Resolution to Be Achieved by the Developed Setup}

Experiments were extended to evaluate the measurement range of the femtosecond laser differential chromatic confocal probe based on the off-axis differential method. In experiments, a target mirror was moved along the Z-direction in a step of $2 \mu \mathrm{m}$ by a piezoelectric (PZT) stage equipped with a capacitance displacement sensor (P-621.2CL, Physik Instrumente, Karlsruhe, Germany). Two confocal outputs were obtained at each Z-position of the target mirror. Figure 12a shows the normalized 
chromatic confocal output $I_{\text {ITLM }}$ obtained through arithmetic operations based on Equation (6) in a wavelength range of $1550-1610 \mathrm{~nm}$. Figure $12 \mathrm{~b}$ shows the variation of the peak wavelength detected from the obtained $I_{\text {ITLM }}$ at each Z-position of the target mirror. The measurement sensitivity, defined as the ratio of the change in the peak wavelength to the Z-displacement of the target mirror, was evaluated to be $-3.233 \mathrm{~nm} / \mu \mathrm{m}$ with good linearity of $R^{2}=0.9996$ over a Z-directional range of $46 \mu \mathrm{m}$. It should be noted that the above results contained the uncertainty that came from the PZT stage employed in the experiments. Meanwhile, the PZT stage had a positioning resolution of $0.2 \mathrm{~nm}$, which was much smaller than that obtained by the femtosecond laser chromatic confocal probe and was enough for the experiments. The verification experiments in this manuscript have been done as the first step of the investigation of the proposed off-axis differential method. Analyses on the measurement uncertainty of the femtosecond laser chromatic confocal probe with the proposed off-axis method will be carried out in future work while considering the positioning uncertainty of the PZT stage.

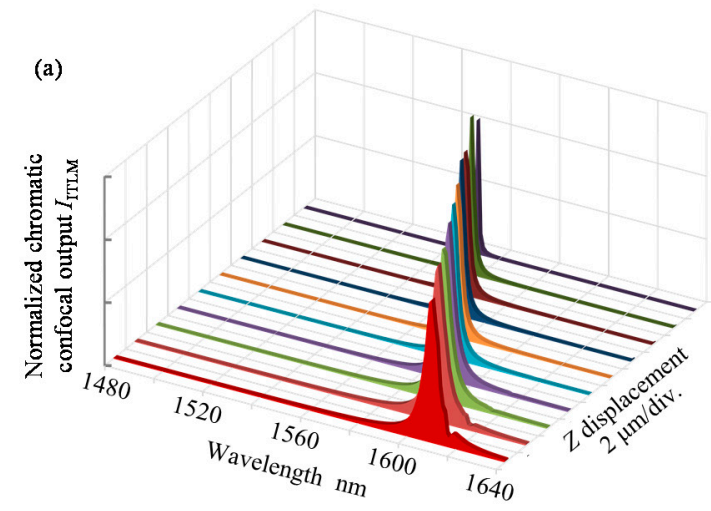

(b)

Figure 12. Experimental results of the wavelength-to-displacement encoding in the proposed off-axis differential chromatic confocal probe system. (a) Normalized chromatic confocal outputs in the range of 1550-1610 nm obtained at each Z-position; (b) variation of the peak wavelength over a range of $46 \mu \mathrm{m}$.

Figure 13 shows the centroid wavelength $\lambda_{\text {ITLM_C }}$ [30] and peak wavelength $\lambda_{\text {ITLM }}$ of $I_{\text {ITLM }}$ encoded from the normalized chromatic confocal output obtained at each Z-position of the target mirror. As can be seen in the figure, the deviations between the peak wavelengths $\lambda_{\text {ITLM }}$ and centroid wavelengths $\lambda_{\text {ITLM_C }}$ of $I_{\text {ITLM }}$ were found to be within $\pm 1.0 \mathrm{~nm}$, which is negligibly small compared with the actual measurement resolution, and is much smaller than that observed in the conventional defocus method (maximum $>40 \mathrm{~nm}$ ) [30]. These results mean that the peak wavelength $\lambda_{\text {ITLM }}$ detected in the normalized chromatic confocal output in the newly proposed off-axis differential method can directly be adopted in the wavelength-to-displacement encoding, without the complex and time-consuming arithmetic operations for obtaining the centroid wavelength $\lambda_{\text {ITLM_C }}$.

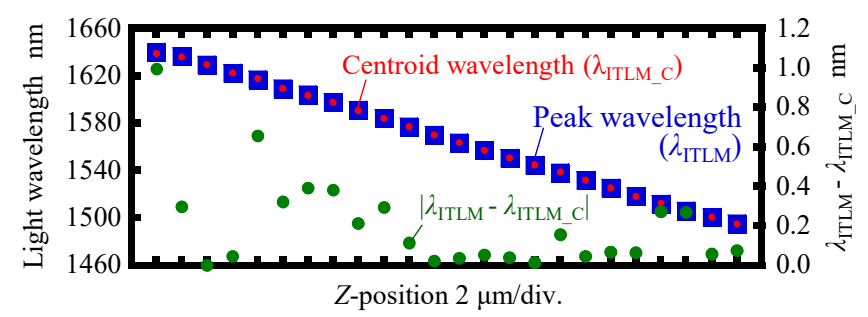

Figure 13. Peak wavelength $\lambda_{\text {ITLM }}$ and centroid wavelength $\lambda_{\text {ITLM_C }}$ of $I_{\text {ITLM }}$ encoded from the normalized chromatic confocal output obtained at each Z-position of the target mirror by the chromatic confocal probe with the proposed off-axis differential method.

Experiments were then carried out to investigate the measurement resolution. In the following experiments, the target mirror was made to move along the Z-direction in a small step by the PZT stage. Figure $14 \mathrm{a}-\mathrm{c}$ show the variation of the detected peak wavelength in the normalized chromatic output 
with a Z-directional step of 40, 30 and $20 \mathrm{~nm}$, respectively. For each moving interval, five repetitive measurements were made, and the mean value from the repeated measurements at each period is plotted in Figure 14. As can be seen in the figures, the change in the peak wavelength with respect to the step Z-displacement given to the target mirror was clearly distinguished in each of the cases. Figure $14 \mathrm{~d}$ shows a standard deviation of the five repetitive measurements at each step observed in the case of Figure 14c. The standard deviation was evaluated to be smaller than $0.14 \mathrm{~nm}$. It should be noted that the above results contained the uncertainty that came from the PZT stage employed in the experiments. Meanwhile, the PZT stage had a positioning resolution of $0.4 \mathrm{~nm}$ and a linearity error of $0.02 \%$ (corresponding to $1.2 \mathrm{~nm}$ regarding a travel range of $6 \mu \mathrm{m}$ in Figure 14c), which were much smaller than those obtained by the femtosecond laser chromatic confocal probe and were enough for the experiments. The verification experiments in this manuscript have been done as the first step of the investigation of the proposed off-axis differential method. Analyses on the measurement uncertainty of the femtosecond laser chromatic confocal probe with the proposed off-axis method will be carried out in future work while considering the positioning uncertainty of the PZT stage.

(a)

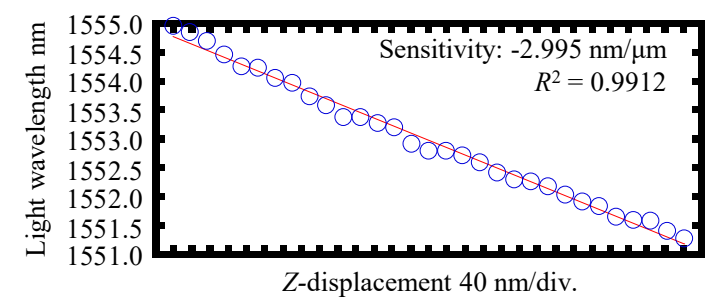

(b)

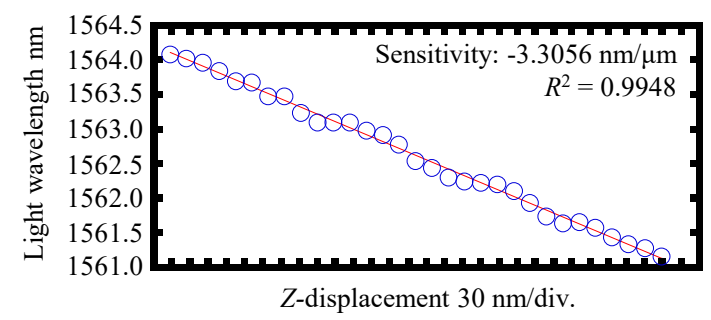

(c)

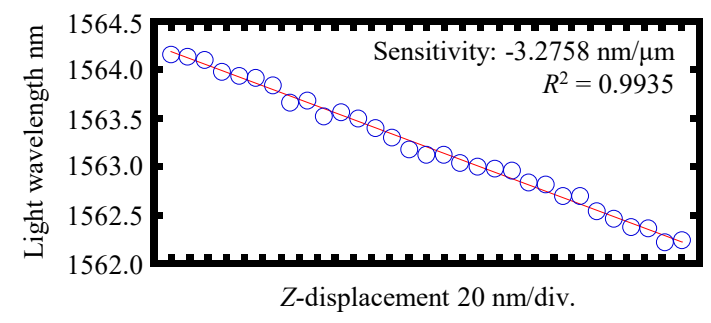

(d)

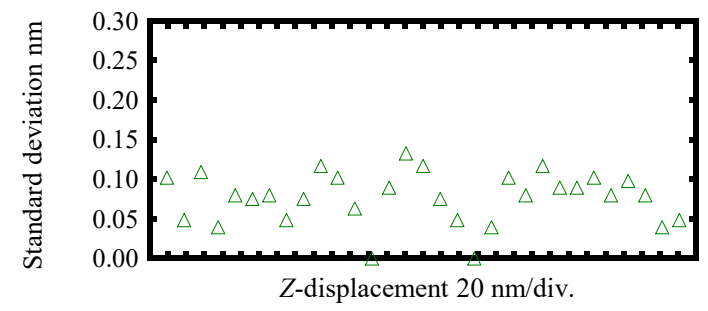

Figure 14. Evaluation of the measurement resolution of the mode-locked femtosecond laser differential chromatic confocal probe with the off-axis differential method. (a) Measured variation of the stage position moved in a step of $40 \mathrm{~nm}$; (b) measured variation of the stage position moved in a step of 30 $\mathrm{nm}$; (c) measured variation of the stage position moved in a step of $20 \mathrm{~nm}$; (d) standard deviation of the repeated measurements in (c). 


\section{Conclusions}

In this paper, an off-axis differential method for a femtosecond laser differential chromatic confocal probe has been proposed to improve the measurement performance, as well as to simplify the optical alignments and the data processing in the peak wavelength- $Z$ position encoding. A major modification has been made to the conventional differential chromatic confocal setup in such a way that the fiber detector in the reference detector unit has been placed to have an offset with respect to the optical axis of the collecting lens in the detector unit, rather than giving a defocus to the fiber detector. This modification enables the newly proposed off-axis setup to obtain a narrower peak having a profile in the normalized chromatic confocal output, as well as to simplify the optical alignment of the differential confocal setup. With the enhancement of the tracking local minimum method, an improved Z-directional measurement range of $46 \mu \mathrm{m}$ has been achieved by the newly proposed off-axis differential method. The possibility of achieving a resolution of $20 \mathrm{~nm}$ has also been verified.

In this paper, attention has been paid to the verification of the feasibility of the newly proposed off-axis method in the femtosecond chromatic confocal probe compared with the conventional setup with the defocus method [27-30]. Further work contains the investigation on the Z-directional measurement resolution with another signal processing algorithm such as the tracking intersection method [30]. It should also be noted that this paper has focused on the proposal of a preliminary idea of an off-axis differential method for the improvement of the signal-to-noise ratio (SNR), the measurement range and measurement resolution of the chromatic confocal probe. A more detailed investigation of the novelty of the displaced probe, as well as the corresponding application in three-dimensional microstructure imaging, will be carried out in future work. Further optimization and simplification of the established differential chromatic confocal probe system for a higher measurement resolution and investigation on the difference between the results of numerical calculations and experimental results described in this paper, as well as the extension of the measurement range by the optimization of the chromatic objective lens, will also be carried out. The application of the developed mode-locked femtosecond chromatic differential confocal probe to the three-dimensional surface profile measurement also remains as a challenge to be addressed and will be carried out in future work.

Author Contributions: Conceptualization, W.G.; methodology, C.C. and R.S.; software, C.C. and R.S.; validation, C.C., H.M. and R.S.; formal analysis, C.C., R.S. and Y.S.; investigation, C.C. and Y.S.; resources, W.G. and Y.S.; Data curation, C.C.; writing-original draft preparation, C.C.; writing-review and editing, Y.S., C.C.; visualization, W.G. and Y.S.; supervision, W.G. and Y.S.; administration, W.G.; funding acquisition, W.G., Y.S. and H.M. All authors have read and agreed to the published version of the manuscript.

Funding: This research was funded by Japan Society for the Promotion of Science (JSPS) 18H01345.

Conflicts of Interest: The authors declare no conflict of interest. The funders had no role in the design of the study; in the collection, analyses, or interpretation of data; in the writing of the manuscript, and in the decision to publish the results.

\section{References}

1. Gao, W.; Kim, S.W.; Bosse, H.; Haitjema, H.; Chen, Y.L.; Lu, X.D.; Knapp, W.; Weckenmann, A.; Estler, W.T.; Kunzmann, H. Measurement technologies for precision positioning. CIRP Ann.-Manuf. Technol. 2015, 64, 773-796. [CrossRef]

2. Gao, W.; Haitjema, H.; Fang, F.Z.; Leach, R.K.; Cheung, C.F.; Savio, E.; Linares, J.M. On-machine and in-process surface metrology for precision manufacturing. CIRP Ann. 2019, 68, 843-866. [CrossRef]

3. Fan, K.C.; Chu, C.L.; Mou, J.I. Development of a low-cost autofocusing probe for profile measurement. Meas. Sci. Technol. 2001, 12, 2137. [CrossRef]

4. Chen, L.C.; Chang, Y.W. Innovative simultaneous confocal full-field 3D surface profilometry for in situ automatic optical inspection (AOI). Meas. Sci. Technol. 2013, 21, 1-12.

5. Chen, L.C.; Chang, Y.W. High accuracy confocal full-field 3-D surface profilometry for micro lenses using a digital fringe projection strategy. Key Eng. Mater. 2008, 364, 113-116. [CrossRef] 
6. Cai, Y.; Yang, B.; Fan, K.C. Robust roll angular error measurement system for precision machines. Opt. Express 2019, 27, 8027-8036. [CrossRef]

7. Stephens, D.J.; Allan, V.J. Light Microscopy Techniques for Live Cell Imaging. Science 2003, 300, 82-86. [CrossRef] [PubMed]

8. Yoneyama, T.; Watanabe, T.; Tamai, S.; Miyashita, K.; Nakada, M. Bright spot analysis for photodynamic diagnosis of brain tumors using confocal microscopy. Photodiagnosis Photodyn. Ther. 2019, 25, 463-471. [CrossRef]

9. Bohn, S.; Sperlich, K.; Allgeier, S.; Bartschat, A.; Prakasam, R.; Reichert, K.M.; Stolz, H.; Guthoff, R.; Mikut, R.; Kohler, B.; et al. Cellular in vivo 3D imaging of the cornea by confocal laser scanning microscopy. Biomed. Opt. Express 2018, 9, 2511-2525. [CrossRef] [PubMed]

10. Hickey, P.C.; Swift, S.R.; Roca, M.G.; Read, N.D. Live-cell imaging of filamentous fungi using vital fluorescent dyes and confocal microscopy. Methods Microbiol. 2004, 34, 63-87.

11. Blateyron, F. Chromatic Confocal Microscopy. In Optical Measurement of Surface Topography; Springer: Berlin/Heidelberg, Germany, 2012; pp. 71-106.

12. Zhao, W.; Jiang, Q.; Qiu, L.; Liu, D. Dual-axes differential confocal microscopy with high axial resolution and long working distance. Opt. Commun. 2011, 284, 15-19. [CrossRef]

13. Lee, C.H.; Wang, J. Noninterferometric differential confocal microscopy with 2-nm depth resolution. Opt. Commun. 1997, 135, 233-237. [CrossRef]

14. Tan, J.; Liu, J.; Wang, Y. Differential confocal microscopy with a wide measuring range based on polychromatic illumination. Meas. Sci. Technol. 2010, 21, 054013. [CrossRef]

15. Sánchez-Ortiga, E.; Sheppard, C.J.; Saavedra, G.; Martínez-Corral, M.; Doblas, A.; Calatayud, A. Subtractive imaging in confocal scanning microscopy using a CCD camera as a detector. Opt. Lett. 2012, 37, 1280-1282. [CrossRef]

16. Qiu, L.; Zhao, W.; Feng, Z.; Ding, X. A lateral super-resolution differential confocal technology with phase-only pupil filter. Optik 2007, 118, 67-73. [CrossRef]

17. Kobayashi, K.; Akiyama, K.; Suzuki, T.; Yoshizawa, I.; Asakura, T. Laser-scanning imaging system for real-time measurements of 3-D object profiles. Opt. Commun. 1989, 74, 165-170. [CrossRef]

18. Butler, D.J.; Horsfall, A.; Hrynevych, M.; Kearney, P.D.; Nugent, K.A. Confocal profilometer with nanometric vertical resolution. Opt. Commun. 1993, 100, 87-92. [CrossRef]

19. Liu, J.; Tan, J.; Bin, H.; Wang, Y. Improved differential confocal microscopy with ultrahigh signal-to-noise ratio and reflectance disturbance resistibility. Appl. Opt. 2009, 48, 6195-6201. [CrossRef]

20. Qiu, L.; Liu, D.; Zhao, W.; Cui, H.; Sheng, Z. Real-time laser differential confocal microscopy without sample reflectivity effects. Opt. Express 2014, 22, 21626-21640. [CrossRef]

21. Zhao, W.; Tan, J.; Qiu, L. Bipolar absolute differential confocal approach to higher spatial resolution. Opt. Express 2004, 12, 5013-5021. [CrossRef]

22. Tan, J.; Wang, F. Theoretical analysis and property study of optical focus detection based on differential confocal microscopy. Meas. Sci. Technol. 2002, 13, 1289. [CrossRef]

23. Zhao, W.; Tan, J.; Qiu, L.; Zou, L. A new laser heterodyne confocal probe for ultraprecision measurement of discontinuous contours. Meas. Sci. Technol. 2005, 16, 497. [CrossRef]

24. Zhao, W.; Tan, J.; Qiu, L. Tri-heterodyne confocal microscope with axial superresolution and higher SNR. Opt. Express 2004, 12, 5191-5197. [CrossRef]

25. Lee, D.R.; Kim, Y.D.; Gweon, D.G.; Yoo, H. Dual-detection confocal fluorescence microscopy: Fluorescence axial imaging without axial scanning. Opt. Express 2013, 21, 17839-17848. [CrossRef] [PubMed]

26. Chen, L.C.; Nguyen, D.T.; Chang, Y.W. Precise optical surface profilometry using innovative chromatic differential confocal microscopy. Opt. Lett. 2016, 41, 5660-5663. [CrossRef] [PubMed]

27. Chen, X.G.; Nakamura, T.; Shimizu, Y.; Chen, C.; Chen, Y.L.; Matsukuma, H.; Gao, W. A chromatic confocal probe with a mode-locked femtosecond laser source. Opt. Laser Tech. 2018, 103, 359-366. [CrossRef]

28. Chen, C.; Sato, R.; Shimizu, Y.; Nakamura, T.; Matsukuma, H.; Gao, W. A Method for Expansion of Z-Directional Measurement Range in a Mode-Locked Femtosecond Laser Chromatic Confocal Probe. Appl. Sci. 2019, 9, 454. [CrossRef]

29. Sato, R.; Shimizu, Y.; Chen, C.; Matsukuma, H.; Gao, W. Investigation and Improvement of Thermal Stability of a Chromatic Confocal Probe with a Mode-Locked Femtosecond Laser Source. Appl. Sci. 2019, 9, 4084. [CrossRef] 
30. Sato, R.; Chen, C.; Matsukuma, H.; Shimizu, Y.; Gao, W. A new signal processing method for a differential chromatic confocal probe with a mode-locked femtosecond laser. Meas. Sci. Technol. 2020, 31, 094004. [CrossRef]

31. Chen, C.; Matsukuma, H.; Sato, R.; Chen, X.; Shimizu, Y.; Gao, W. Theoretical investigation on measurement range of a femtosecond laser chromatic confocal probe by utilizing side-lobe of axial response. In Proceedings of the 2018 IEEE International Conference on Advanced Manufacturing (ICAM), Yunlin, Taiwan, 16-18 November 2018; pp. 362-364.

32. Gu, M. Principles of Three Dimensional Imaging in Confocal Microscopes; World Scientific: Singapore, 1996; pp. 117-220.

33. Cox, I.J.; Sheppard, C.J.; Wilson, T. Improvement in resolution by nearly confocal microscopy. Appl. Opt. 1982, 21, 778-781. [CrossRef]

34. Pawley, J. (Ed.) Handbook of Biological Confocal Microscopy; Springer Science \& Business Media: Berlin, Germany, 2006; Volume 236, pp. 17-20.

35. Wilson, T.; Sheppard, C. Theory and Practice of Scanning Optical Microscopy; Academic Press: London, UK, 1984; pp. 17-20.

36. Wilson, T.; Carlini, A.R. Size of the detector in confocal imaging systems. Opt. Lett. 1987, 12, $227-229$. [CrossRef] [PubMed]

37. Kimura, S.; Wilson, T. Confocal scanning optical microscope using single-mode fiber for signal detection. Appl. Opt. 1991, 30, 2143-2149. [CrossRef] [PubMed]

38. Barrell, K.F.; Pask, C. Optical fibre excitation by lenses. Opt. Acta Int. J. Opt. 1979, 26, 91-108. [CrossRef]

Publisher's Note: MDPI stays neutral with regard to jurisdictional claims in published maps and institutional affiliations. 